\title{
Kalman Filter for Spinning Spacecraft Attitude Estimation
}

\author{
F. Landis Markley ${ }^{\mathrm{a}}$ \\ NASA Goddard Space Flight Center, Greenbelt, MD 20771 \\ and \\ Joseph E. Sedlak ${ }^{\mathrm{b}}$ \\ a.i. solutions, Inc., Lanham, MD 20706
}

\begin{abstract}
This paper presents a Kalman filter using a seven-component attitude state vector comprising the angular momentum components in an inertial reference frame, the angular momentum components in the body frame, and a rotation angle. The relatively slow variation of these parameters makes this parameterization advantageous for spinning spacecraft attitude estimation. The filter accounts for the constraint that the magnitude of the angular momentum vector is the same in the inertial and body frames by employing a reduced six-component error state. Four variants of the filter, defined by different choices for the reduced error state, are tested against a quaternion-based filter using simulated data for the THEMIS mission. Three of these variants choose three of the components of the error state to be the infinitesimal attitude error angles, facilitating the computation of measurement sensitivity matrices and causing the usual $3 \times 3$ attitude covariance matrix to be a submatrix of the $6 \times 6$ covariance of the error state. These variants differ in their choice for the other three components of the error state. The variant employing the infinitesimal attitude error angles and the angular momentum components in an inertial reference frame as the error state shows the best combination of robustness and efficiency in the simulations. Attitude estimation results using THEMIS flight data are also presented.
\end{abstract}

\section{Introduction}

Attitude estimation is often more difficult for spinning spacecraft than for three-axis stabilized spacecraft. The parameters representing the spacecraft attitude and its time rate of change vary more rapidly in the spinning case, and gyro rate measurements are often lacking, requiring Euler's equations for modeling the attitude dynamics. This paper uses a seven-parameter angular-momentum-based representation that is advantageous for this application. ${ }^{1}$ The seven state vector elements are the angular momentum components in an inertial reference frame, the angular momentum components in the spacecraft's body frame, and a rotation angle. These parameters are subject to the constraint that the magnitude of the angular momentum vector is the same in the inertial and body frames.

We have developed a series of extended Kalman filters (EKFs) employing this representation, to which we give the generic name SpinKF. The constraint on the state vector allows us to employ a six-component error state instead of the error vector of the full seven-component state, in parallel with the procedure commonly used to estimate the constrained four-component quaternion representation of attitude. ${ }^{2}$ The conceptual advantages of this dimensional reduction, as more truly representing the actual degrees of freedom of the system, have been debated at length, ${ }^{3-6}$ but the computational advantages are indisputable. The different versions of SpinKF all use the same seven-component state but differ in their specification of the six-component error state. The first, SpinKF $1,{ }^{7}$ was incorporated into the attitude ground support system for spinning spacecraft at the NASA Goddard Space Flight Center and used for operational support of the Space Technology 5 (ST5) mission. ${ }^{8-10}$ SpinKF2, introduced in Ref. 10, has been used to support the Time History of Events and Macroscale Interaction during Substorms (THEMIS) mission. " This paper introduces two new implementations, SpinKF3 and SpinKF4. In all the versions except SpinKF1, three of the components of the state error vector are the usual infinitesimal attitude error angles, making the usual $3 \times 3$ attitude covariance matrix a submatrix of the $6 \times 6$ covariance and greatly facilitating the computation of measurement sensitivity matrices.

\footnotetext{
aerospace Engineer, Guidance, Navigation, and Control Systems Engineering Branch, Code 591, AIAA Fellow

${ }^{b}$ Senior Engineer, 10001 Derekwood Lane
} 
The paper first presents the seven-parameter angular-momentum-based representation. This is followed by a brief introduction to SpinKF2 and complete derivations of SpinKF3 and SpinKF4. Data simulated to represent the THEMIS spacecraft are used to compare the performance of the four SpinKF variants and a more conventional EKF based on the quaternion and body rotation rate. Attitude estimation results using THEMIS flight data are presented and conclusions are drawn from the results of the simulations and flight data results.

\section{Angular-Momentum-Based Attitude Parameterization}

The spacecraft's angular momentum about its center of mass expressed in its body frame, $\mathbf{L}_{B}$, or expressed in an inertial reference frame, $L_{I}$, obeys the equations of motion ${ }^{12}$

$$
d \mathbf{L}_{B} / d t=\mathbf{N}_{B}-\omega_{B I} \times \mathbf{L}_{B} \quad \text { and } \quad d \mathbf{L}_{I} / d t=\mathbf{N}_{I}=A_{B I}^{T} \mathbf{N}_{B}
$$

as well as the constraint

$$
\left|\mathbf{L}_{B}\right|=\left|\mathbf{L}_{l}\right| \equiv L,
$$

where $\mathbf{N}$ is the external torque, $A_{B I}$ is the inertial-to-body attitude matrix, and the angular velocity $\omega_{B I}$ is given by

$$
\boldsymbol{\omega}_{B I}=J^{-1}\left(\mathbf{L}_{B}-\mathbf{L}_{i n t}\right)
$$

In this equation $J$ is the spacecraft moment of inertia tensor and $\mathbf{L}_{\text {int }}$ is the angular momentum of any moving parts (reaction wheels, steerable antennas or solar arrays, flexible modes, fuel slosh, etc.) relative to the spacecraft. Spacecraft dynamics are commonly modeled by Eq. (1a) and kinematics by

$$
d A_{B I} / d t=-\left[\boldsymbol{\omega}_{B I} \times\right] A_{B I},
$$

where

$$
[\mathrm{v} \times] \equiv\left[\begin{array}{ccc}
0 & -v_{3} & v_{2} \\
v_{3} & 0 & -v_{1} \\
-v_{2} & v_{1} & 0
\end{array}\right]
$$

denotes the cross product matrix for an arbitrary 3-component vector $\mathbf{v}$. A quaternion or some other lowerdimensional representation of $A_{B I}$ is often integrated rather than Eq. (4), but this distinction is not important for this paper. An alternative formulation uses Eq. (1b) and

$$
\mathbf{L}_{B}=A_{B I} \mathbf{L}_{I}
$$

in place of Eq. (1a). Both of these formulations have the disadvantage for application to spinning spacecraft that many components of the state vector are rapidly varying parameters, requiring small integration steps.

The formulation in this paper is based on the observation that both the attitude matrix $A_{B I}$ and the rotation matrix

$$
R_{B I}=\left(\hat{\mathbf{L}}_{B} \cdot \hat{\mathbf{L}}_{I}\right) I_{3 \times 3}-\hat{\mathbf{L}}_{I} \hat{\mathbf{L}}_{B}^{T}+\hat{\mathbf{L}}_{B} \hat{\mathbf{L}}_{I}^{T}+\left(1+\hat{\mathbf{L}}_{B} \cdot \hat{\mathbf{L}}_{I}\right)^{-1}\left(\hat{\mathbf{L}}_{B} \times \hat{\mathbf{L}}_{I}\right)\left(\hat{\mathbf{L}}_{B} \times \hat{\mathbf{L}}_{I}\right)^{T}
$$

take the unit vector $\hat{\mathbf{L}}_{I} \equiv \mathrm{L}_{I} / L$ to $\hat{\mathbf{L}}_{B} \equiv \mathbf{L}_{B} / L$. Thus the product $R_{B I}^{T} A_{B I}$ takes $\hat{\mathbf{L}}_{I}$ into itself, so it is a rotation about $\hat{\mathbf{L}}_{I}$ and can be expressed as

$$
R_{B I}^{T} A_{B I}=R\left(\hat{\mathbf{L}}_{I}, \zeta\right)
$$

where

$$
R(\mathbf{e}, \phi) \equiv(\cos \phi) I_{3 \times 3}+(1-\cos \phi) \mathrm{ee}^{T}-\sin \phi[\mathrm{e} \times]
$$


is the matrix representing a rotation by angle $\phi$ about axis e. Multiplying both sides of Eq. (8a) by $R_{B I}$ gives

$$
A_{B I}=R_{B I} R\left(\hat{\mathbf{L}}_{I}, \zeta\right)
$$

Multiplying this equation on the right by $I_{3 \times 3}=R_{B I}^{T} R_{B I}$ and using the identity $R_{B I} R\left(\hat{\mathbf{L}}_{I}, \zeta\right) R_{B I}^{T}=R\left(\hat{\mathbf{L}}_{B}, \zeta\right)$ gives

$$
A_{B I}=R\left(\hat{\mathbf{L}}_{B}, \zeta\right) R_{B I},
$$

Either Eq. (8a) or (8b) gives our parameterization of the attitude matrix $A_{B I}(\mathbf{x})$ in terms of the seven-component state vector

$$
\mathbf{x}=\left[\begin{array}{lll}
\mathbf{L}_{B}^{T} & \mathbf{L}_{I}^{T} & \zeta
\end{array}\right]^{T}
$$

The dynamics are given by Eqs. (1a), (1b), and a differential equation for $\zeta$, derived in Ref. 1 and as Eq. (21) below.

\section{Reduction to a Six-Component Error State}

A straightforward Kalman filter implementation would use the $7 \times 7$ covariance $P_{x} \equiv E\left\{(\Delta \mathbf{x})(\Delta \mathbf{x})^{T}\right\}$ of the error vector $\Delta \mathbf{x} \equiv \mathbf{x}-\overline{\mathbf{x}}$, where $\overline{\mathbf{x}} \equiv E\{\mathbf{x}\}$ is the expectation of $\mathbf{x}$. As pointed out in Ref. 7, the constraint of Eq. (2) means that the covariance can be represented by a matrix of rank six, in the same way that the unity norm constraint of the four-component quaternion representation leads to a rank-deficient covariance matrix. ${ }^{2}$ In the present case, the covariance matrix is assumed to have a null vector $\mathbf{x}_{\text {null }}$,

$$
P_{x} \mathbf{x}_{\text {null }}=0_{7 \times 1},
$$

that is orthogonal to all the vectors representing physically possible errors in $\mathbf{x}$. The six physically possible errors are a variation in $\zeta$, two independent variations of $\mathbf{L}_{B}$ perpendicular to $\overline{\mathbf{L}}_{B}$, two variations of $\mathbf{L}_{I}$ perpendicular to $\overline{\mathbf{L}}_{I}$, and simultaneous length-changing equal-magnitude variations of $\mathbf{L}_{B}$ in the direction of $\overline{\mathbf{L}}_{B}$ and of $\mathbf{L}_{I}$ in the direction of $\overline{\mathbf{L}}_{l}$. Thus the normalized null eigenvector of $P_{x}$ must be

$$
\mathbf{x}_{\text {null }}= \pm \frac{1}{\sqrt{2}}\left[\begin{array}{lll}
\hat{\mathbf{L}}_{B}^{T} & -\hat{\mathbf{L}}_{I}^{T} & 0
\end{array}\right]^{T},
$$

which is a vector of errors violating the norm constraint of Eq. (2).

We seek a $7 \times 6$ matrix $S$ with a Moore-Penrose pseudoinverse ${ }^{13} S^{+}$such that

$$
S^{+} S=I_{6 \times 6}, S S^{+}=I_{7 \times 7}-\mathbf{x}_{n u l l} \mathbf{x}_{\text {null }}^{T} \text {, and } S^{+} \mathbf{x}_{\text {null }}=0_{6 \times 1} .
$$

Note that $S$ and $S^{+}$must be functions of expectations rather than true values, which are unknown by the estimator. The six-dimensional error vector

$$
\Delta \mathbf{y} \equiv S^{+} \Delta \mathbf{x}
$$

has a nonsingular $6 \times 6$ error covariance $P_{y}$ given by

$$
P_{y} \equiv E\left\{(\Delta \mathbf{y})(\Delta \mathbf{y})^{T}\right\}=S^{+} P_{x}\left(S^{+}\right)^{T} .
$$

Because of Eqs. (10) and (12b), the $7 \times 7$ error covariance $P_{x}$ can be recovered by

$$
S P_{y} S^{T}=S S^{+} P_{x}\left(S^{+}\right)^{T} S^{T}=S S^{+} P_{x}\left(S S^{+}\right)^{T}=P_{x}
$$

and the seven-component error vector, which must be orthogonal to $\mathbf{x}_{\text {null }}$, by

$$
\Delta \mathrm{x}=I_{7 \times 7} \Delta \mathrm{x}=\left(S S^{+}+\mathrm{x}_{n u l l} \mathrm{x}_{\text {mull }}^{T}\right) \Delta \mathrm{x}=S \Delta \mathbf{y}
$$


Beginning with SpinKF2, the matrix $S^{+}$has been chosen so that the first three components of $\Delta y$ are equal to the three attitude error angles in the body reference frame, $\Delta \theta$. This choice results in the upper left-hand corner of $P_{y}$ being the usual $3 \times 3$ attitude error covariance, and we will see later that it also greatly facilitates computation of measurement sensitivity matrices. SpinKF1 used a different formulation, the details of which can be found in Ref. 7.

The expression for $\Delta \theta$ as a function of $\Delta \mathrm{x}$ is found by computing the first-order increment $\Delta A_{B I}$ of $A_{B I}(\mathrm{x})$ with variations $\Delta \mathrm{L}_{B}, \Delta \mathrm{L}_{I}$, and $\Delta \zeta$, and using the relationship

$$
[\Delta \theta \times]=-\left(\Delta A_{B I}\right) A_{B I}^{T}
$$

This gives, after considerable algebra,

$$
\Delta \boldsymbol{\theta}=-L^{-1} \hat{\mathbf{L}}_{B} \times\left[\Delta \mathbf{L}_{B}-A_{B I}(\mathbf{x}) \Delta \mathbf{L}_{I}\right]+\left[\Delta \zeta-L^{-1} \mathbf{w} \cdot\left(\Delta \mathbf{L}_{B}+\Delta \mathbf{L}_{I}\right)\right] \hat{\mathbf{L}}_{B},
$$

where

$$
\mathbf{w} \equiv\left(1+\hat{\mathbf{L}}_{B} \cdot \hat{\mathbf{L}}_{I}\right)^{-1}\left(\hat{\mathbf{L}}_{B} \times \hat{\mathbf{L}}_{I}\right)=\left(L^{2}+\mathbf{L}_{B} \cdot \mathbf{L}_{I}\right)^{-1}\left(\mathbf{L}_{B} \times \mathbf{L}_{I}\right)
$$

Interpreting Eq. (18) as a relation of time variations of $\boldsymbol{\theta}, \mathbf{L}_{B}, \mathbf{L}_{I}$, and $\zeta$ gives

$$
\boldsymbol{\omega}_{B I}=-L^{-1} \hat{\mathbf{L}}_{B} \times\left[\frac{d \mathbf{L}_{B}}{d t}-A_{B I}(\mathbf{x}) \frac{d \mathbf{L}_{I}}{d t}\right]+\left[\frac{d \zeta}{d t}-L^{-1} \mathbf{w} \cdot\left(\frac{d \mathbf{L}_{B}}{d t}+\frac{d \mathbf{L}_{I}}{d t}\right)\right] \hat{\mathbf{L}}_{B} .
$$

After substituting Eq. (1), Eq. (20) reduces to the dynamic equation for $\zeta$,

$$
d \zeta / d t=\left(1+\hat{\mathbf{L}}_{B} \cdot \hat{\mathbf{L}}_{I}\right)^{-1}\left[\left(\hat{\mathbf{L}}_{B}+\hat{\mathbf{L}}_{I}\right) \cdot \boldsymbol{\omega}_{B I}+L^{-1}\left(\hat{\mathbf{L}}_{B} \times \hat{\mathbf{L}}_{I}\right) \cdot\left(\mathbf{N}_{B}+\mathbf{N}_{I}\right)\right]
$$

The upper three rows of $S^{+}$are given by Eq. (18). The different varieties of SpinKF are distinguished by different choices for the lower three rows.

\section{A. SpinKF2}

SpinKF2 satisfies Eqs. (12) by choosing the lower three rows of $S^{+}$to give

$$
S_{2}^{+}=\left[\begin{array}{ccc}
\bar{L}^{-1}\left\{-\left[\hat{\overline{\mathbf{L}}}_{B} \times\right]-\hat{\overline{\mathbf{L}}}_{B} \overline{\mathbf{w}}^{T}\right\} & \bar{L}^{-1}\left\{\left[\hat{\mathbf{L}}_{B} \times\right] A_{B I}(\overline{\mathbf{x}})-\hat{\overline{\mathbf{L}}}_{B} \overline{\mathbf{w}}^{T}\right\} & \hat{\overline{\mathbf{L}}}_{B} \\
I_{3 \times 3} & A_{B I}(\overline{\mathbf{x}}) & 0_{3 \times 1}
\end{array}\right]
$$

so Eq. (13) gives

$$
\Delta \mathbf{y}_{2}=\left[\begin{array}{c}
\Delta \theta \\
\Delta \mathbf{L}_{B}+A_{B I}(\overline{\mathbf{x}}) \Delta \mathbf{L}_{I}
\end{array}\right]
$$

Note our convention of using a numerical subscript $n$ to indicate that an expression is valid only for a specific SpinKF $n$ algorithm. The appearance of $A_{B I}(\overline{\mathbf{x}})$ in $\triangle \mathbf{y}$ made SpinKF2 more complex than SpinKF3 and SpinKF4, so we will not present its further development here. The interested reader can find the details in Ref. 10.

\section{B. SpinKF3}

SpinKF3 chooses the lower three rows of $S^{+}$to give

$$
S_{3}^{+}=\left[\begin{array}{ccc}
\bar{L}^{-1}\left\{-\left[\hat{\overline{\mathbf{L}}}_{B} \times\right]-\hat{\overline{\mathbf{L}}}_{B} \overline{\mathbf{w}}^{T}\right\} & \bar{L}^{-1}\left\{\left[\hat{\overline{\mathbf{L}}}_{B} \times\right] A_{B I}(\overline{\mathbf{x}})-\hat{\overline{\mathbf{L}}}_{B} \overline{\mathbf{w}}^{T}\right\} & \hat{\overline{\mathbf{L}}}_{B} \\
I_{3 \times 3}-\frac{1}{2} \hat{\mathbf{L}}_{B} \hat{\mathbf{L}}_{B}^{T} & \frac{1}{2} \hat{\mathbf{L}}_{B} \hat{\mathbf{L}}_{I}^{T} & 0_{3 \times 1}
\end{array}\right] .
$$


This means that the six-component error state vector is

$$
\Delta \mathbf{y}_{3}=\left[\begin{array}{c}
\Delta \theta \\
\Delta \mathbf{L}_{B}-\frac{1}{2}\left(\hat{\mathbf{L}}_{B} \cdot \Delta \mathbf{L}_{B}-\hat{\overline{\mathbf{L}}}_{I} \cdot \Delta \mathbf{L}_{I}\right) \hat{\overline{\mathbf{L}}}_{B}
\end{array}\right]=\left[\begin{array}{c}
\Delta \theta \\
\Delta \mathbf{L}_{B}
\end{array}\right]
$$

because $\hat{\overline{\mathbf{L}}}_{B} \cdot \Delta \mathrm{L}_{B}=\Delta L=\hat{\overline{\mathrm{L}}}_{I} \cdot \Delta \mathbf{L} I$ for state errors that obey the angular momentum norm constraint. This $\Delta \mathbf{y}$ is the same as would be obtained in an EKF for a four-component quaternion and $\mathbf{L}_{B}$ by using the techniques in Ref. 2 to handle the quaternion norm constraint. The pseudoinverse of $S_{3}^{+}$is

$$
S_{3}=\left[\begin{array}{cc}
0_{3 \times 3} & I_{3 \times 3} \\
-A_{B I}^{T}(\overline{\mathbf{x}})\left[\overline{\mathbf{L}}_{B} \times\right] & A_{B I}^{T}(\overline{\mathbf{x}}) \\
\left.\hat{\mathbf{L}}_{B}+\hat{\mathbf{L}}_{B} \times A_{B I}(\overline{\mathbf{x}}) \overline{\mathbf{w}}\right]^{T} & \bar{L}^{-1}\left[\overline{\mathbf{w}}+A_{B I}(\overline{\mathbf{x}}) \overline{\mathbf{w}}\right]^{T}
\end{array}\right] .
$$

\section{SpinKF4}

SpinKF4 chooses the lower three rows of $S^{+}$to give

$$
S_{4}^{+}=\left[\begin{array}{ccc}
\bar{L}^{-1}\left\{-\left[\hat{\overline{\mathbf{L}}}_{B} \times\right]-\hat{\overline{\mathbf{L}}}_{B} \overline{\mathbf{w}}^{T}\right\} & \bar{L}^{-1}\left\{\left[\hat{\overline{\mathbf{L}}}_{B} \times\right] A_{B I}(\overline{\mathbf{x}})-\hat{\overline{\mathbf{L}}}_{B} \overline{\mathbf{W}}^{T}\right\} & \hat{\overline{\mathbf{L}}}_{B} \\
\frac{1}{2} \hat{\overline{\mathbf{L}}}_{I} \hat{\mathbf{L}}_{B}^{T} & I_{3 \times 3}-\frac{1}{2} \hat{\overline{\mathbf{L}}}_{I} \hat{\overline{\mathbf{L}}}_{I}^{T} & 0_{3 \times 1}
\end{array}\right] .
$$

and therefore

$$
\Delta \mathbf{y}_{4}=\left[\begin{array}{c}
\Delta \boldsymbol{\theta} \\
\Delta \mathbf{L}_{I}+\frac{1}{2}\left(\hat{\overline{\mathbf{L}}}_{B} \cdot \Delta \mathbf{L}_{B}-\hat{\mathbf{L}}_{I} \cdot \Delta \mathbf{L}_{I}\right) \hat{\mathbf{L}}_{I}
\end{array}\right]=\left[\begin{array}{c}
\Delta \theta \\
\Delta \mathbf{L}_{I}
\end{array}\right] .
$$

The. motivation for SpinKF4 is that $\Delta \mathbf{L}_{I}$ varies more slowly than $\Delta \mathbf{L}_{B}$, which should make the covariance propagation easier. The pseudoinverse of $S_{4}^{+}$is

$$
S_{4}=\left[\begin{array}{cc}
{\left[\overline{\mathbf{L}}_{B} \times\right]} & A_{B I}(\overline{\mathbf{x}}) \\
0_{3 \times 3} & I_{3 \times 3} \\
\left(\hat{\overline{\mathbf{L}}}_{B}-\hat{\overline{\mathrm{L}}}_{B} \times \overline{\mathbf{w}}\right)^{T} & \bar{L}^{-1}\left[\overline{\mathbf{w}}+A_{B I}^{T}(\overline{\mathbf{x}}) \overline{\mathbf{w}}\right]^{T}
\end{array}\right] .
$$

\section{Kalman Filter Formulation}

A Kalman filter for the seven-component state vector $\mathbf{x}$ uses Eqs. (1) and (21) to propagate the state estimate between observations. The filter update for a measurement $z=h(x)$ is given by ${ }^{14}$

$$
\mathbf{x}(+)=\mathbf{x}(-)+K_{x}[\tilde{\mathbf{z}}-\mathbf{h}(-)],
$$

where the arguments $(-)$ and $(+)$ denote estimates before and after the update, respectively, $\tilde{\mathbf{z}}$ denotes the measured value, $\mathbf{h}(-) \equiv \mathbf{h}(\mathbf{x}(-))$, and $K_{x}$ is the Kalman gain. The gain is given by

$$
K_{x}=P_{x}(-) H_{x}^{T}\left[H_{x} P_{x}(-) H_{x}^{T}+R\right]^{-1},
$$

where the measurement sensitivity matrix is

$$
H_{x} \equiv \partial \mathbf{h}(\mathbf{x}) / \partial \mathbf{x}
$$

and $R$ is the measurement error covariance. The covariance is updated by

$$
P_{x}(+)=\left(I_{7 \times 7}-K_{x} H_{x}\right) P_{x}(-),
$$


To avoid using a singular covariance matrix, we substitute Eq. (15) into Eq. (31), giving

$$
K_{x}=S K_{y},
$$

where $K_{y}$ is given by

$$
K_{y}=P_{y}(-) H_{y}^{T}\left[H_{y} P_{y}(-) H_{y}^{T}+R\right]^{-1}
$$

with

$$
H_{y} \equiv H_{x} S=[\partial \mathbf{h}(\mathbf{x}) / \partial \mathbf{x}](\partial \mathbf{x} / \partial \mathbf{y})=\partial \mathbf{h} / \partial \mathbf{y}
$$

The matrices $S$ and $S^{+}$are always assumed to be evaluated with the pre-update estimates. Substituting Eq. (15) into Eq. (33) and using Eqs. (12a) and (12b) gives

$$
P_{y}(+)=\left(I_{6 \times 6}-K_{y} H_{y}\right) P_{y}(-),
$$

\section{A. State Update: SpinKF3}

The state update in SpinKF3 is given by

$$
\mathbf{x}(+)=\mathbf{x}(-)+S_{3} K_{y}[\tilde{\mathbf{z}}-\mathbf{h}(-)] \equiv \mathbf{x}(-)+S_{3}\left[\begin{array}{c}
\Delta \bar{\theta} \\
\Delta \overline{\mathbf{L}}_{B}
\end{array}\right] .
$$

Specifically,

$$
\begin{gathered}
\overline{\mathbf{L}}_{B}(+)=\overline{\mathbf{L}}_{B}(-)+\Delta \overline{\mathbf{L}}_{B} \\
\overline{\mathbf{L}}_{I}(+)=\overline{\mathbf{L}}_{I}(-)+A_{B I}^{T}(-)\left[\Delta \overline{\mathbf{L}}_{B}+\Delta \overline{\boldsymbol{\theta}} \times \overline{\mathbf{L}}_{B}(-)\right]=A_{B I}^{T}(-)\left[\overline{\mathbf{L}}_{B}(-)+\Delta \overline{\mathbf{L}}_{B}+\Delta \overline{\boldsymbol{\theta}} \times \overline{\mathbf{L}}_{B}(-)\right] .
\end{gathered}
$$

The update preserves the norm constraint of Eq. (2) to first order but violates it in second order, in parallel with the quaternion case. ${ }^{2}$ The cross-product term in Eq. (39b) appears to be the first-order approximation to a rotation, so we trust the magnitude of $\overline{\mathbf{L}}_{B}(+)$ more than that of $\overline{\mathbf{L}}_{I}(+)$ and renormalize the latter by

$$
\overline{\mathbf{L}}_{I}(++)=\left|\overline{\mathbf{L}}_{B}(+)\right| \hat{\mathbf{L}}_{I}(+)
$$

to restore the norm constraint.

\section{B. State Update: SpinKF4}

The state update in SpinKF4 is given by

$$
\mathbf{x}(+)=\mathbf{x}(-)+S_{4} K_{y}[\tilde{\mathbf{z}}-\mathbf{h}(-)] \equiv \mathbf{x}(-)+S_{4}\left[\begin{array}{c}
\Delta \bar{\theta} \\
\Delta \overline{\mathbf{L}}_{I}
\end{array}\right] .
$$

Specifically,

$$
\begin{gathered}
\overline{\mathbf{L}}_{B}(+)=\overline{\mathbf{L}}_{B}(-)-\Delta \overline{\boldsymbol{\theta}} \times \overline{\mathbf{L}}_{B}(-)+A_{B I}(-) \Delta \overline{\mathbf{L}}_{I}=A_{B I}(-)\left[\overline{\mathbf{L}}_{I}(-)+\Delta \overline{\mathbf{L}}_{I}\right]-\Delta \overline{\mathbf{\theta}} \times \overline{\mathbf{L}}_{B}(-) \\
\overline{\mathbf{L}}_{I}(+)=\overline{\mathbf{L}}_{I}(-)+\Delta \overline{\mathbf{L}}_{I} .
\end{gathered}
$$

Since the cross-product term occurs in Eq. (42a), the norm constraint in SpinKF4 is restored by

$$
\overline{\mathbf{L}}_{B}(++)=\left|\overline{\mathbf{L}}_{I}(+)\right| \hat{\mathbf{L}}_{B}(+) \text {. }
$$

\section{Covariance Propagation}

The matrices $F_{y}$ and $G_{y}$ in the covariance propagation equation

$$
d P_{y} / d t=F_{y} P_{y}+P_{y} F_{y}^{T}+G_{y} Q G_{y}^{T}
$$


are most easily computed directly from the equations for the reduced state vector $y$. Thus, neither $S^{+}$nor the $7 \times 7$ covariance matrix $P_{x}$ appears in our Kalman filter, and $S$ appears only in the state update.

We assume that

$$
\mathbf{N}_{B}=\overline{\mathbf{N}}_{B}+J \mathbf{n}_{u} \text { and } \mathbf{L}_{i n t}=\overline{\mathbf{L}}_{i n t}-J \mathbf{n}_{\mathbf{v}},
$$

where $\mathbf{n}_{u}$ and $\mathbf{n}_{v}$ are assumed to be independent zero-mean Gaussian white noise processes with

$$
E\left\{\left[\begin{array}{l}
\mathbf{n}_{v}(t) \\
\mathbf{n}_{u}(t)
\end{array}\right]\left[\begin{array}{l}
\mathbf{n}_{v}\left(t^{\prime}\right) \\
\mathbf{n}_{u}\left(t^{\prime}\right)
\end{array}\right]^{T}\right\}=Q(t) \delta\left(t-t^{\prime}\right)=\left[\begin{array}{cc}
Q_{v}(t) & 0_{3 \times 3} \\
0_{3 \times 3} & Q_{u}(t)
\end{array}\right] \delta\left(t-t^{\prime}\right),
$$

where $\delta\left(t-t^{\prime}\right)$ is the Dirac delta function. The definitions in Eq. (45) are arranged so that $\mathbf{n}_{u}$ and $\mathbf{n}_{v}$ have dimensions of angular acceleration and angular velocity, respectively, and $Q_{u}$ and $Q_{v}$ have units of $\mathrm{rad}^{2} / \mathrm{sec}^{3}$ and $\mathrm{rad}^{2} / \mathrm{sec}$. Note that $Q_{v}$ may be non-zero even for a nominally rigid spacecraft.

To first order, the attitude error vector obeys the dynamics equation ${ }^{15}$

$$
d(\Delta \boldsymbol{\theta}) / d t=\Delta \boldsymbol{\omega}_{B I}-\overline{\boldsymbol{\omega}}_{B I} \times \Delta \boldsymbol{\theta}
$$

For SpinKF3 we need the dynamics equation obeyed by the angular momentum error in the body frame

$$
d\left(\Delta \mathbf{L}_{B}\right) / d t=\Delta\left[\mathbf{N}_{B}-\boldsymbol{\omega}_{B I} \times \mathbf{L}_{B}\right]=\overline{\mathbf{L}}_{B} \times \Delta \boldsymbol{\omega}_{B I}-\overline{\boldsymbol{\omega}}_{B I} \times \Delta \mathbf{L}_{B}+J \mathbf{n}_{u} .
$$

From Eq. (3) we have

$$
\Delta \boldsymbol{\omega}_{B I}=J^{-1}\left(\Delta \mathbf{L}_{B}-\Delta \mathbf{L}_{i n t}\right)=J^{-1} \Delta \mathbf{L}_{B}+\mathbf{n}_{v},
$$

so differentiating Eq. (25) gives

$$
\frac{d\left(\Delta \mathbf{y}_{3}\right)}{d t}=F_{y 3} \Delta \mathbf{y}_{3}+G_{y 3}\left[\begin{array}{l}
\mathbf{n}_{v} \\
\mathbf{n}_{u}
\end{array}\right]
$$

with

$$
F_{y 3} \equiv\left[\begin{array}{cc}
-\left[\overline{\boldsymbol{\omega}}_{B I} \times\right] & J^{-1} \\
0_{3 \times 3} & {\left[\overline{\mathbf{L}}_{B} \times\right] J^{-1}-\left[\overline{\boldsymbol{\omega}}_{B I} \times\right]}
\end{array}\right] \quad \text { and } \quad G_{y 3} \equiv\left[\begin{array}{cc}
I_{3 \times 3} & 0_{3 \times 3} \\
{\left[\overline{\mathbf{L}}_{B} \times\right]} & J
\end{array}\right] \text {. }
$$

It is well known that the solution of Eq. (44) can be written as

$$
P_{y}(t)=\Phi\left(t, t_{0}\right) P_{y}\left(t_{0}\right) \Phi^{T}\left(t, t_{0}\right)+\int_{t_{0}}^{t} \Phi\left(t, t^{\prime}\right) G_{y} Q\left(t^{\prime}\right) G_{y}^{T} \Phi^{T}\left(t, t^{\prime}\right) d t^{\prime}
$$

where the transition matrix $\Phi\left(t, t_{0}\right)$ is a solution of $d \Phi\left(t, t_{0}\right) / d t=F_{y}(t) \Phi\left(t, t_{0}\right)$ with initial value $\Phi\left(t_{0}, t_{0}\right)=I_{6 \times 6}$. Using Eq. (4), we can show that in SpinKF3

$$
\Phi_{3}\left(t, t_{0}\right)=\left[\begin{array}{cc}
A_{B I}(t) A_{B I}^{T}\left(t_{0}\right) & \Phi_{\theta L 3}\left(t, t_{0}\right) \\
0_{3 \times 3} & \Phi_{L L 3}\left(t, t_{0}\right)
\end{array}\right],
$$

where $\Phi_{L L 3}\left(t, t_{0}\right)$ is a solution of

$$
d \Phi_{L L 3}\left(t, t_{0}\right) / d t=\left\{\left[\overline{\mathbf{L}}_{B}(t) \times\right] J^{-1}-\left[\overline{\boldsymbol{\omega}}_{B I}(t) \times\right]\right\} \Phi_{L L 3}\left(t, t_{0}\right)
$$


with initial value $\Phi_{L L 3}\left(t_{0}, t_{0}\right)=I_{3 \times 3}$, and

$$
\Phi_{\theta L 3}\left(t, t_{0}\right)=A_{B I}(t) \int_{t_{0}}^{t} A_{B I}^{T}\left(t^{\prime}\right) J^{-1} \Phi_{L L 3}\left(t^{\prime}, t_{0}\right) d t^{\prime} .
$$

SpinKF4 uses the dynamics equation obeyed by the angular momentum error in the inertial frame

$$
\begin{aligned}
d\left(\Delta \mathbf{L}_{I}\right) / d t=\Delta \mathbf{N}_{I}=\Delta\left[A_{B I}^{T}(\mathbf{x}) \mathbf{N}_{B}\right] & =\Delta\left\{A_{B I}^{T}(\overline{\mathbf{x}})\left(I_{3 \times 3}+[\Delta \boldsymbol{\theta} \times]\right)\left(\overline{\mathbf{N}}_{B}+J \mathbf{n}_{u}\right)\right\} \\
& =A_{B I}^{T}(\overline{\mathbf{x}})\left(J \mathbf{n}_{u}-\overline{\mathbf{N}}_{B} \times \Delta \boldsymbol{\theta}\right) .
\end{aligned}
$$

We also use Eqs. (3) and (17) to obtain

$$
\begin{aligned}
\Delta \boldsymbol{\omega}_{B I}=J^{-1} \Delta\left[A_{B I}(\mathbf{x}) \mathbf{L}_{I}-\mathbf{L}_{i n t}\right] & =J^{-1}\left\{-[\Delta \theta \times] A_{B I}(\overline{\mathbf{x}}) \overline{\mathbf{L}}_{I}+A_{B I}(\overline{\mathbf{x}}) \Delta \mathbf{L}_{I}-\Delta \mathbf{L}_{i n t}\right\} \\
& =J^{-1}\left[\overline{\mathbf{L}}_{B} \times \Delta \theta+A_{B I}(\overline{\mathbf{x}}) \Delta \mathbf{L}_{I}\right]+\mathbf{n}_{v} .
\end{aligned}
$$

An equation analogous to Eq. (50) is obtained for SpinKF4 with

$$
F_{y 4} \equiv\left[\begin{array}{cc}
J^{-1}\left[\overline{\mathbf{L}}_{B} \times\right]-\left[\overline{\mathbf{\omega}}_{B I} \times\right] & J^{-1} A_{B I}(\overline{\mathbf{x}}) \\
-A_{B I}^{T}(\overline{\mathbf{x}})\left[\overline{\mathbf{N}}_{B} \times\right] & 0_{3 \times 3}
\end{array}\right] \quad \text { and } \quad G_{y 4} \equiv\left[\begin{array}{cc}
I_{3 \times 3} & 0_{3 \times 3} \\
0_{3 \times 3} & A_{B I}^{T}(\overline{\mathbf{x}}) J
\end{array}\right] \text {. }
$$

There is a convenient expression for the state transition matrix $\Phi_{4}\left(t, t_{0}\right)$ in the torque-free case, $\overline{\mathbf{N}}_{B}=0_{3 \times 1}$, but not in the general case.

\section{Filter Implementation}

SpinKF has been implemented in MATLAB ${ }^{\mathrm{TM}}$ as a subsystem of the Multimission Spin-Axis Stabilized Spacecraft Attitude Ground Support System that has supported NASA Goddard Space Flight Center missions for many years. The new EKF subsystem adds the capability to solve for a time-dependent attitude history and could be used for real-time applications, if needed. The software processes sensor data and presents it to the EKF as vector observations. After discarding outliers, the EKF integrates the state vector and its covariance to the next observation time using a $4^{\text {th }}$-order Runge-Kutta integrator with an appropriate time step. The EKF obtains the spacecraft ephemeris and geomagnetic field and computes torques due to gravity gradients and any residual constant spacecraft magnetization at each integration step. ${ }^{12}$ Control torques, if known, can be included. Then the sensor residual and the sensitivity matrix are computed, and the state and covariance are updated using the symmetric Joseph form ${ }^{14}$ for the covariance update in place of Eq. (37).

\section{A. Singula rity Avoidance}

It is clear throughout the development of this filter that the spacecraft angular momentum is required to be nonzero, and that the algorithm is also singular when $\mathbf{L}_{B}$ and $\mathbf{L}_{I}$ are $180^{\circ}$ apart. The software checks for the latter singular condition and redefines the inertial reference frame so that $\mathbf{L}_{l}$ is always greater than a user-specified distance from $-\mathbf{L}_{B}$ in the modified frame, transforming all reference vectors along with $\mathbf{L}_{l}$. Reconstructing the attitude referenced to the standard inertial frame is only a matter of keeping track of these reference frame rotations, which are all handled internally and are totally transparent to the user of the software.

\section{B. Measurement Models}

It is easier to calculate the measurement sensitivity matrices directly from the six-component error vector $y$ than from the seven-component state vector $\mathbf{x}$. For a vector measurement, $\mathbf{z}=\mathbf{v}_{B}$ and

$$
\mathbf{h}(\mathbf{x})=A_{B I}(\mathbf{x}) \mathrm{v}_{I} \approx\left(I_{3 \times 3}-[\Delta \theta \times]\right) A_{B I}(\overline{\mathbf{x}}) \mathrm{v}_{I}=\overline{\mathbf{v}}_{B}+\overline{\mathrm{v}}_{B} \times \Delta \theta,
$$

where

$$
\overline{\mathbf{v}}_{B} \equiv A_{B I}(\overline{\mathbf{x}}) \mathbf{v}_{I}
$$


It follows from Eq. (25) and the rightmost part of Eq. (36) that

$$
H_{y}^{\text {vector }}=\left[\begin{array}{ll}
{\left[\overline{\mathbf{v}}_{B} \times\right]} & 0_{3 \times 3}
\end{array}\right] \text {. }
$$

In the case of quaternion measurements, such as those output from an autonomous star tracker, an error quaternion is defined by

$$
\Delta q=\left[\Delta \mathbf{q}^{T} \Delta q_{4}\right]^{T}=q(-) \otimes q_{o b s}^{-1},
$$

where $q(-)$ is constructed from the pre-update state $\mathbf{x}(-)$, and where the quaternion multiplication convention of Ref. 2 is used, so that $A\left(q \otimes q^{\prime}\right)=A(q) A\left(q^{\prime}\right)$. Then the observation residual is given by

$$
\tilde{\mathbf{z}}-\mathbf{h}(-) \equiv-2 \operatorname{sign}\left(\Delta q_{4}\right) \frac{\sin ^{-1}(|\Delta \mathbf{q}|)}{|\Delta \mathbf{q}|} \Delta \mathbf{q}
$$

and the measurement sensitivity matrix is simply

$$
H_{y}^{\text {quatemion }}=\left[\begin{array}{ll}
I_{3 \times 3} & 0_{3 \times 3}
\end{array}\right] .
$$

For spacecraft with gyros, the gyro outputs are used as measurements to update the state, rather than being used for state propagation in model replacement mode. ${ }^{2}$ The gyro sensitivity matrix is the only measurement sensitivity matrix that is different between SpinKF3 and SpinKF4. The gyro measurement model is

$$
\mathbf{h}(\mathbf{x})=U J^{-1}\left(\mathbf{L}_{B}-\mathbf{L}_{i n t}\right),
$$

where the rows of $U$ are the gyro sensitive axes in the body frame. Thus in SpinKF3 we have simply

$$
H_{y 3}^{g y r o}=U\left[0_{3 \times 3} \quad J^{-1}\right]
$$

In SpinKF4, on the other hand, $H_{x}^{\text {gyro }}=U\left[\begin{array}{lll}J^{-1} & 0_{3 \times 3} & 0_{3 \times 1}\end{array}\right]$, which yields

$$
H_{y 4}^{\text {gyro }}=H_{x}^{\text {gyro }} S_{4}=U J^{-1}\left[\left[\overline{\mathbf{L}}_{B} \times\right] \quad A_{B I}(\overline{\mathbf{x}})\right] .
$$

\section{Testing with Simulated Data}

Several tests were performed to compare the performance of different versions of SpinKF and the Unit Vector Filter $^{16}$ (UVF) that has supported many three-axis stabilized spacecraft at Goddard Space Flight Center over the past 16 years. For application to spinning spacecraft, the UVF was modified to estimate the quaternion and rotation rate rather than the gyro biases, to use dynamics propagation rather than gyro propagation, and to use linearized dynamics matrices for covariance propagation that are related to that of SpinKF3 by

$$
F_{U V F}=\left[\begin{array}{cc}
I_{3 \times 3} & 0_{3 \times 3} \\
0 & J \times 3
\end{array}\right]^{-1} F_{y 3}\left[\begin{array}{cc}
I_{3 \times 3} & 0_{3 \times 3} \\
0 & J \times 3
\end{array}\right] \text { and } \quad G_{U V F}=\left[\begin{array}{cc}
I_{3 \times 3} & 0_{3 \times 3} \\
0 & J \times 3
\end{array}\right]^{-1} G_{y 3} .
$$

\section{A. Simulation Parameters}

The simulator allows modeling of spinning spacecraft with a wide variety of sensor types and several torque scenarios. The tests exercised all the key features of the filters using simulation parameters based on the THEMIS series of spinning spacecraft. ${ }^{11}$ THEMIS consists of five identical probes designed to study magnetic substorms in the Earth's magnetosphere. The probes will be maneuvered into orbits with periods and phasing that cause them to line up radially from the Earth at apogee once every four days. Apogee will occur in the magnetotail during the first part of the primary science campaign from December 2007 to April 2008. 
THEMIS was launched on February 17, 2007. The initial orbit for all five probes had an inclination of about $14^{\circ}$, period of 31 hours, and eccentricity of 0.85 . The perigee and apogee heights were roughly $900 \mathrm{~km}$ and $87000 \mathrm{~km}$, respectively. The spin axes have all since been oriented along the normal to the ecliptic plane, but the simulations use the initial release spin direction that had a right ascension of $51.3^{\circ}$ and declination of $-23.2^{\circ}$.

The probes are low mass $(126 \mathrm{~kg})$, spin-stabilized at 20 revolutions per minute (rpm) about the body $Z$-axis, and carry single-head slit-type Sun sensors and three-axis magnetometers (TAM) for attitude determination. The slit Sun sensor measures the Sun angle from the $Z$-axis once per spin and generates a timing pulse. The ground software constructs a body frame Sun vector from the measured angle and the known slit azimuth. The TAM measurement frequency used for attitude determination is $8 \mathrm{~Hz}$, which gives a generous 24 observations per spin period.

The TAM is both an attitude sensor and a science instrument, and its inherent noise is very small $(0.01 \mathrm{nT})$. Thus, the TAM error for attitude determination is almost entirely due to errors in the reference field, which were modeled in the simulation and in the filters as Gaussian errors with standard deviation of $100 \mathrm{nT}$ on each axis. The International Geomagnetic Reference Field (IGRF) was used to model the magnetic field. The Sun sensor errors are due to a combination of Sun angle measurement error and Sun pulse timing error. They were modeled as Gaussian errors with standard deviation of $0.16^{\circ}$ per axis.

The inertia tensor used for all the simulations is

$$
J=\operatorname{diag}([13,13,22]) \mathrm{kg}-\mathrm{m}^{2}
$$

This value is of the correct magnitude for THEMIS after deployment of the TAM boom but prior to deployment of the long wire radial booms. The tensor was taken to be axisymmetric to simplify computation of simulated attitudes. A nutation angle of $2^{\circ}$ was assumed in all the torque-free runs. The simulator and the filter propagation routine allow for environmental torques, but these were turned off for the tests presented here.

The process noise was taken to be

$$
Q_{v}=10^{-6} \mathrm{rad}^{2} / \mathrm{sec} \times \operatorname{diag}([1,1,3])
$$

and

$$
Q_{u}=10^{-7} \mathrm{rad}^{2} / \mathrm{sec}^{3} \times \operatorname{diag}([1,1,3])
$$

The initial state covariance matrix was constructed assuming an initial attitude uncertainty of $20^{\circ}$ about $X$ and $Y$ and $180^{\circ}$ about $Z$ and a rate uncertainty of $10 \mathrm{deg} / \mathrm{sec}$ on each axis.

The simulation time span is 25 minutes and is centered on perigee where the altitude is low enough for the TAM data to be useful. The first three minutes of each run are discarded from the results to allow time for the filter transients to damp out.

\section{B. Test Results}

The values reported in this section are a measure of the spin axis pointing error, which is the root-sum-square of the $X$ - and $Y$-axis errors. The spin phase error is not included since this is much less important operationally. The $X$ - and $Y$-errors are obtained by converting the filter state vector estimates into a history of equivalent quaternions, comparing with the truth model quaternions, and determining the root-mean-square error about each axis.

A series of tests were designed to stress the filters in four different ways: reducing the number of observations per spin period, increasing the propagation step size, increasing the initial attitude error, and increasing the error in the inertia tensor model.

Prior to these stressing cases, four baseline scenarios were tested to verify filter performance with parameters similar to those used for operational support of THEMIS. The Nominal scenario consists of torque-free motion, a $2^{\circ}$ nutation angle, no sensor misalignments, an accurately modeled inertia tensor, and uses TAM data (24 per spin period) and Sun sensor data (one per spin period). The second scenario was the Nominal scenario with gyro data added. The third scenario was the same as the Nominal scenario, but with misalignments of $0.1^{\circ}$ on the TAM and Sun sensor. The fourth scenario was also similar to the Nominal, but with a torque applied in the body frame for $10 \%$ of each spin period to precess the spin axis by $30^{\circ}$. Note that the filter is NOT given the torque values for any of the tests presented here; if the torque values are passed to the filter, the filter will agree very closely with the truth model (the errors then are indistinguishable from the nominal case). 
Table 1 shows the results of these first tests for SpinKF1, 2, 3, 4, and the modified UVF described by Eqs. (68a,b). In these tests, the initial attitude errors relative to the truth model are $10^{\circ}$ about the $X$ - and $Y$-axes, $45^{\circ}$ about $Z$, and the rate errors are $5 \mathrm{deg} / \mathrm{sec}$ about $X$ and $Y$, and $10 \mathrm{deg} / \mathrm{sec}$ about $Z$.

Table 1. Results of baseline filter tests.

\begin{tabular}{|l|l|l|l|l|l|}
\hline Scenario & $\begin{array}{l}\text { SpinKF1 } \\
\text { Pointing Error }\end{array}$ & $\begin{array}{l}\text { SpinKF2 } \\
\text { Pointing Error }\end{array}$ & $\begin{array}{l}\text { SpinKF3 } \\
\text { Pointing Error }\end{array}$ & $\begin{array}{l}\text { SpinKF4 } \\
\text { Pointing Error }\end{array}$ & $\begin{array}{l}\text { UVF } \\
\text { Pointing Error }\end{array}$ \\
\hline Nominal & $0.0810^{\circ}$ & $0.0873^{\circ}$ & $0.0873^{\circ}$ & $0.0874^{\circ}$ & $0.0873^{\circ}$ \\
\hline Nominal plus gyro & $0.0769^{\circ}$ & $0.0249^{\circ}$ & $0.0249^{\circ}$ & $0.0249^{\circ}$ & $0.0249^{\circ}$ \\
\hline Misaligned sensors & $0.1325^{\circ}$ & $0.1388^{\circ}$ & $0.1387^{\circ}$ & $0.1390^{\circ}$ & $0.1392^{\circ}$ \\
\hline $\begin{array}{l}30^{\circ} \text { Slew } \\
\text { (no torque data to KF) }\end{array}$ & $0.1142^{\circ}$ & $0.7224^{\circ}$ & $0.7139^{\circ}$ & $0.7348^{\circ}$ & $0.7110^{\circ}$ \\
\hline
\end{tabular}

It is clear from Table 1 that all five filters are performing well and are in good agreement. As a measure of computational burden, the mean clock times for these runs are 47, 29, 25, 25, and 26 sec for SpinKF1, SpinKF2, SpinKF3, SpinKF4, and the UVF, respectively. These times are platform-dependent, but the relative times are significant. The SpinKF1 is slower in part because its partial derivative expressions are much more complicated than the other filters, but also because its MATLAB ${ }^{\mathrm{TM}}$ code has not been as thoroughly optimized. It is not clear why SpinKF1 performs better than the other filters with the attitude slew here and in the next example.

The next set of tests examines how the filters respond with reduced data availability. Table 2 shows results for the same four scenarios as in Table 1, but with the TAM data frequency reduced from 24 to 2.1 observations per spin period. (The number is deliberately nonintegral so the spin phase at the TAM measurement times will not be constant.) The propagation step size was set to $1 / 8$ second to match the step size in the baseline tests (driven in that case by the TAM frequency).

Table 2. Filter test results with TAM data frequency reduced to 2.1 observations per spin period.

\begin{tabular}{|l|l|l|l|l|l|}
\hline Scenario & $\begin{array}{l}\text { SpinKF1 } \\
\text { Pointing Error }\end{array}$ & $\begin{array}{l}\text { SpinKF2 } \\
\text { Pointing Error }\end{array}$ & $\begin{array}{l}\text { SpinKF3 } \\
\text { Pointing Error }\end{array}$ & $\begin{array}{l}\text { SpinKF4 } \\
\text { Pointing Error }\end{array}$ & $\begin{array}{l}\text { UVF } \\
\text { Pointing Error }\end{array}$ \\
\hline Nominal & $0.1171^{\circ}$ & Diverged & $0.1097^{\circ}$ & $0.1098^{\circ}$ & $0.1097^{\circ}$ \\
\hline Nominal plus gyro & $0.1120^{\circ}$ & $0.0364^{\circ}$ & $0.0367^{\circ}$ & $0.0370^{\circ}$ & $0.0367^{\circ}$ \\
\hline Misaligned sensors & $0.1596^{\circ}$ & Diverged & $0.1538^{\circ}$ & $0.1537^{\circ}$ & $0.1538^{\circ}$ \\
\hline $\begin{array}{l}30^{\circ} \text { Slew } \\
\text { (no torque data to KF) }\end{array}$ & $0.1625^{\circ}$ & $1.2726^{\circ}$ & $1.2662^{\circ}$ & $1.2654^{\circ}$ & $1.2764^{\circ}$ \\
\hline
\end{tabular}

With fewer data, the attitude errors shown in Table 2 are larger than those in Table 1, but with the exception of SpinKF2, the filters still perform well. When the TAM frequency is reduced further, all the filters tend to diverge, depending largely on the chance transients from the first few data points.

The filter determines the propagation step size first, from the data time steps, and second, from an input parameter that specifies a maximum allowed time step. Whenever the time between observations is larger than this maximum step size, additional integration steps are inserted as required. To test the numerical propagation, the next test increased the maximum allowed step size while keeping the actual data frequency low. With the TAM frequency at 2.1 observations per spin period and the maximum step size equal to $0.5 \mathrm{sec}$, all the filters except SpinKF1 and SpinKF4 begin to fail the test scenarios. Table 3 shows that increasing the step size further to 0.7 sec causes SpinKF4 to fail in some cases, but displays no clear pattern. The convergence region is small for all the filters when the data are sparse and the propagation step size is large. Whether a filter diverges or not then is largely just a happenstance caused by the first few data points. If the transients push the state far enough from the truth so that nonlinearities in the sensor models become important, the filter often never recovers. When the step size is increased to $1 \mathrm{sec}$, SpinKF1 also diverges. 
Table 3. Filter test results with TAM data frequency reduced to 2.1 observations per spin period and the maximum propagation step size increased to $0.7 \mathrm{sec}$.

\begin{tabular}{|l|l|l|l|l|l|}
\hline Scenario & $\begin{array}{l}\text { SpinKF1 } \\
\text { Pointing Error }\end{array}$ & $\begin{array}{l}\text { SpinKF2 } \\
\text { Pointing Error }\end{array}$ & $\begin{array}{l}\text { SpinKF3 } \\
\text { Pointing Error }\end{array}$ & $\begin{array}{l}\text { SpinKF4 } \\
\text { Pointing Error }\end{array}$ & $\begin{array}{l}\text { UVF } \\
\text { Pointing Error }\end{array}$ \\
\hline Nominal & $0.7027^{\circ}$ & Diverged & Diverged & Diverged & Diverged \\
\hline Misaligned sensors & $0.7115^{\circ}$ & Diverged & Diverged & $0.1816^{\circ}$ & Diverged \\
\hline $\begin{array}{l}30^{\circ} \text { Slew } \\
\text { (no torque data to KF) }\end{array}$ & $0.1631^{\circ}$ & Diverged & $1.4762^{\circ}$ & $1.2690^{\circ}$ & Diverged \\
\hline
\end{tabular}

The next series of tests considers the effect of varying the initial attitude error in the nominal scenario (torque-free, $2^{\circ}$ nutation, $8 \mathrm{~Hz}$ TAM, no misalignments, $1 / 8 \mathrm{sec}$ propagation step size). The initial rate error is kept at $5 \mathrm{deg} / \mathrm{sec}$ about $X$ and $Y$, and $10 \mathrm{deg} / \mathrm{sec}$ about $Z$. Table 4 shows selected results.

Table 4. Filter results for nominal scenario with various initial attitude errors.

\begin{tabular}{|l|l|l|l|l|l|}
\hline Initial Attitude Errors & $\begin{array}{l}\text { SpinKF1 } \\
\text { Pointing } \\
\text { Error }\end{array}$ & $\begin{array}{l}\text { SpinKF2 } \\
\text { Pointing } \\
\text { Error }\end{array}$ & $\begin{array}{l}\text { SpinKF3 } \\
\text { Pointing } \\
\text { Error }\end{array}$ & $\begin{array}{l}\text { SpinKF4 } \\
\text { Pointing } \\
\text { Error }\end{array}$ & $\begin{array}{l}\text { UVF } \\
\text { Pointing } \\
\text { Error }\end{array}$ \\
\hline Xerr $=30^{\circ}$, Yerr $=30^{\circ}$, Zerr $=0$ & $0.0802^{\circ}$ & $0.0873^{\circ}$ & $0.0873^{\circ}$ & $0.0874^{\circ}$ & $0.0873^{\circ}$ \\
\hline Xerr $=60^{\circ}$, Yerr $=60^{\circ}$, Zerr $=0$ & $0.0802^{\circ}$ & $0.0873^{\circ}$ & $3.1932^{\circ}$ & $0.1119^{\circ}$ & $0.0873^{\circ}$ \\
\hline Xerr $=80^{\circ}$, Yerr $=80^{\circ}$, Zerr $=0$ & Diverged & $0.0873^{\circ}$ & $14.4281^{\circ}$ & Diverged & $0.1199^{\circ}$ \\
\hline Xerr $=90^{\circ}$, Yerr $=0$, Zerr $=0$ & $0.0808^{\circ}$ & $0.0873^{\circ}$ & $0.0873^{\circ}$ & $0.0874^{\circ}$ & $0.0873^{\circ}$ \\
\hline Xerr $=-90^{\circ}$, Yerr $=0$, Zerr $=0$ & $0.0810^{\circ}$ & $16.9711^{\circ}$ & $0.0891^{\circ}$ & $0.0874^{\circ}$ & $0.0873^{\circ}$ \\
\hline Xerr $=120^{\circ}$, Yerr $=0$, Zerr $=0$ & $0.0810^{\circ}$ & $2.2748^{\circ}$ & Diverged & $0.0875^{\circ}$ & $0.0874^{\circ}$ \\
\hline Xerr $=0$, Yerr $=120^{\circ}$, Zerr $=0$ & Diverged & Diverged & Diverged & Diverged & Diverged \\
\hline Xerr $=170^{\circ}$, Yerr $=0$, Zerr $=0$ & $11.2017^{\circ}$ & Diverged & $0.0873^{\circ}$ & Diverged & Diverged \\
\hline Xerr $=-170^{\circ}$, Yerr $=0$, Zerr $=0$ & $3.7612^{\circ}$ & Diverged & Diverged & $0.0876^{\circ}$ & Diverged \\
\hline Xerr $=30^{\circ}$, Yerr $=30^{\circ}$, Zerr $=-175^{\circ}$ & $0.0804^{\circ}$ & $16.9142^{\circ}$ & $0.0833^{\circ}$ & Diverged & $0.0815^{\circ}$ \\
\hline Xerr $=0$, Yerr $=0$, Zerr $=175^{\circ}$ & $0.0807^{\circ}$ & $0.0890^{\circ}$ & $0.0875^{\circ}$ & $0.0881^{\circ}$ & $0.0873^{\circ}$ \\
\hline
\end{tabular}

Entries in Table 4 with relatively large values indicate cases where the filter did converge but took longer than three minutes to settle down to linear behavior, so some of the initial transients are included in the statistics. The results are mixed, but it is clear the SpinKF filters do not outperform the UVF when presented with large initial errors.

The last row in Table 4 shows that all five filters converged when the $X$ and $Y$ errors were zero and the $Z$ error was large, that is, with spin phase error only. The initial spin phase typically is completely unknown. Experience with many runs with simulations and actual flight data shows that occasional filter divergence can be expected even when the initial pointing direction is known to within a few degrees. When this happens, restarting the filter with the phase shifted by $180^{\circ}$ almost always gives a good solution. To avoid these occasional unnecessary divergences, the filter could self-initialize by making a rough attitude estimate using data from the first few spin periods. This feature should be added to the operational version of the filter in a future release.

The final set of tests is again based on the nominal scenario, but now includes various amounts of error in the inertia tensor. The simulator uses the inertia given by Eq. 69 in all cases, but the filter here is given an incorrect inertia for propagation. The first row in Table 5 uses an inertia tensor close to the actual value for THEMIS after TAM boom 
deployment. In this case, all the filters except SpinKF1 show similar errors of only $1.1^{\circ}$. The errors are relatively small here because the inertia tensor error consists primarily of a rotation of the principal axis frame about the nominal body frame $Z$-axis by about $41^{\circ}$ with only $0.15^{\circ} \mathrm{X}$ and $Y$ rotation. The $41^{\circ} Z$ rotation has no significant effect since the truth model inertia tensor is axisymmetric. The combined $X$ and $Y$ rotation tips the major principal axis away from the body $Z$-axis, which induces coning motion into the propagation that is not present in the truth model attitude. However, a larger source of error in this example is that this tensor is far from axially symmetric, so the nutational motion is not predicted accurately. The eigenvalues of $J$ are approximately $7.8,18.5$, and $21.7 \mathrm{~kg}-\mathrm{m}^{2}$; the lack of symmetry is indicated by the difference between the first and second eigenvalues not being small relative to the third.

Table 5. Filter results for nominal scenario with various errors in the inertia tensor.

\begin{tabular}{|c|c|c|c|c|c|}
\hline $\begin{array}{l}\text { Inertia Tensor } \\
\left(\mathrm{kg}-\mathrm{m}^{2}\right)\end{array}$ & $\begin{array}{l}\text { SpinKF1 } \\
\text { Pointing } \\
\text { Error }\end{array}$ & $\begin{array}{l}\text { SpinKF2 } \\
\text { Pointing } \\
\text { Error }\end{array}$ & $\begin{array}{l}\text { SpinkF3 } \\
\text { Pointing } \\
\text { Error }\end{array}$ & $\begin{array}{l}\text { SpinKF4 } \\
\text { Pointing } \\
\text { Error }\end{array}$ & $\begin{array}{l}\text { UVF } \\
\text { Pointing } \\
\text { Error }\end{array}$ \\
\hline$J_{E K F}=\left[\begin{array}{ccc}12.4 & -5.3 & -.02 \\
-5.3 & 14.0 & -.01 \\
-.02 & -.01 & 21.7\end{array}\right]$ & $1.9872^{\circ}$ & $1.1143^{\circ}$ & $1.1134^{\circ}$ & $1.1164^{\circ}$ & $1.1137^{\circ}$ \\
\hline$J_{E K F}=\left[\begin{array}{ccc}12.4 & -2 & -1 \\
-2 & 14.0 & 0 \\
-1 & 0 & 21.7\end{array}\right]$ & $7.4997^{\circ}$ & $6.5419^{\circ}$ & $7.0813^{\circ}$ & $6.8028^{\circ}$ & $7.3736^{\circ}$ \\
\hline$J_{E K F}=\left[\begin{array}{ccc}14 & 0 & -1.5 \\
0 & 12 & 0 \\
-1.5 & 0 & 22\end{array}\right]$ & $11.9418^{\circ}$ & $10.3834^{\circ}$ & $14.5196^{\circ}$ & $10.5923^{\circ}$ & $13.7981^{\circ}$ \\
\hline$J_{E K F}=\left[\begin{array}{ccc}14 & -2 & -2 \\
-2 & 12 & 0 \\
-2 & 0 & 22\end{array}\right]$ & $15.9104^{\circ}$ & $17.8481^{\circ}$ & $30.1164^{\circ}$ & $13.9457^{\circ}$ & $38.6044^{\circ}$ \\
\hline
\end{tabular}

In the second case in Table 5, the principal axis frame is tipped by $6.6^{\circ}$ from the body frame. Compared with the first case, the symmetry error is less important since the eigenvalues are $11.0,15.3$, and $21.8 \mathrm{~kg}-\mathrm{m}^{2}$. Thus, the coning error predominates. In the third and fourth cases in Table 5, the principal axis frame is tipped by $10.3^{\circ}$ and $14.1^{\circ}$, respectively, and again, coning error predominates. As the coning angle increases, the SpinKF4 error follows it closely, but the other filters begin to show larger errors. The covariance propagation is probably more accurate for SpinKF4 since it uses $\Delta \mathbf{L}_{I}$ rather than $\Delta \mathbf{L}_{B}$ in its error state vector. Specifically, the lower half of $F_{y 4}$ in Eq. (58a) does not include a factor of $J$, so this part of the covariance propagation is not corrupted by the error in the inertia tensor.

\section{Estimation Results with Flight Data}

The SpinKF2 version of the filter has been used for ground attitude support for the five THEMIS probes. Before filter performance could be assessed, the sensors needed to be calibrated. In particular, the TAM was deployed after launch on a 2-meter boom and has an alignment uncertainty of approximately $1^{\circ}$. The calibration utility uses an attitude-independent method ${ }^{17}$ to determine biases, scale factors, and skewness of the axes, and an iterative method ${ }^{18}$ to determine the orthogonal sensor alignment. SpinKF2 is a key part of the iterative method. The calibrations were repeated several times for each probe using data sets from March, 2007. Each data span covers 50 to 60 minutes near perigee. The reference magnetic field was obtained using the IGRF 2005 model to $10^{\text {th }}$ order along with definitive ephemerides for the spacecraft positions.

The spacecraft attitude history was determined using SpinKF2 before and after calibration. Before calibration, the sensor residuals from the filter show offsets of $1^{\circ}$ to $2^{\circ}$ from zero primarily due to misalignment. Calibration removes these offsets and reduces their standard deviations by up to a factor of two. 
The spin vector is nominally aligned with the body $Z$-axis and is constant in an inertial frame; however, a nonzero coning angle is expected, especially prior to deployment of the radial wire booms. The calibration reduces the standard deviation of the $Z$-axis pointing direction by roughly a factor of two, yielding a post-calibration 3-sigma $Z$ axis uncertainty of $0.4^{\circ}$ to $0.5^{\circ}$ for Probes A-D and $0.7^{\circ}$ for Probe $\mathrm{E}$. This is in excellent agreement with the $Z$-axis coning motion predicted from the inertia tensors. The angular momentum direction is more nearly constant; it does not vary due to coning. The angular momentum direction 3-sigma uncertainties range from less than $0.1^{\circ}$ to $0.3^{\circ}$ for all post-calibration solutions for the five probes.

Figure 1 shows a typical solution for the estimated angular momentum right ascension, declination, and magnitude for Probe A. The variation in the angular momentum direction seen in Figure 1 is not physical. Gravity gradient is the largest environmental torque, and it precesses the angular momentum vector by only $0.01^{\circ}$ per orbit, much less than the variation seen in Figure 1. It is likely that this small variation is due to error in the reference field caused by a small, but not entirely negligible, ephemeris error. This problem was seen, greatly exaggerated, early in the mission before accurate ephemerides were available. On the other hand, the apparent slow downward drift of the angular momentum magnitude results from cooling of onboard fuel that, being forced to the outer edge of the tanks, contracts away from the spin axis, thereby increasing the moment of inertia about that axis and decreasing the spin rate. Assuming a constant inertia tensor, the filter misinterprets this as a decreasing angular momentum.
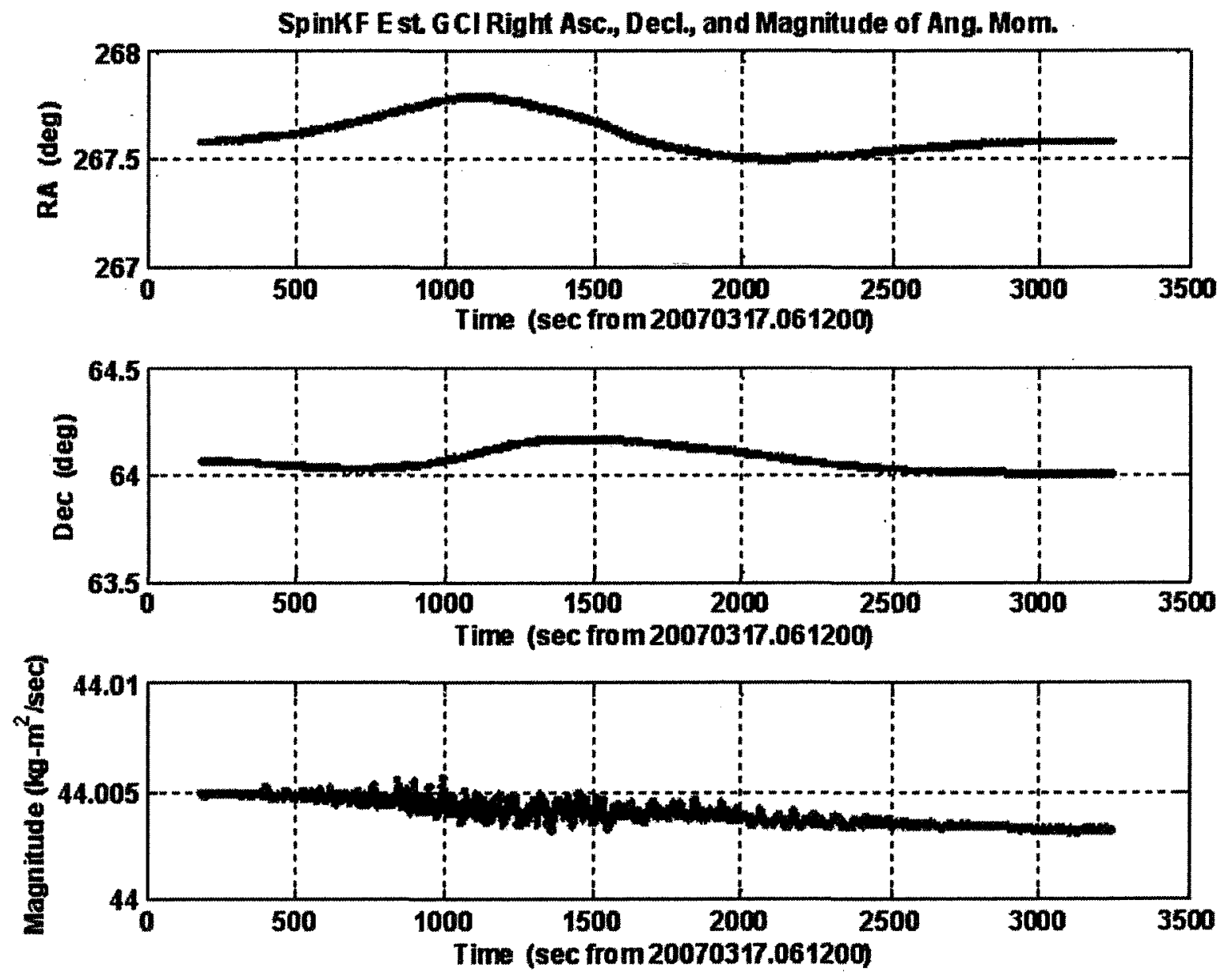

Fig. 1. Right ascension, declination, and magnitude of the angular momentum vector estimated using SpinKF2 for THEMIS Probe A after calibration of the magnetometer. 


\section{Conclusions}

A class of extended Kalman filters for spinning spacecraft has been developed using a seven-component angularmomentum-based attitude parameterization. The state vector is constrained by the requirement that the magnitude of the angular momentum is the same in all reference frames. This constraint allows the filters to employ a sixcomponent error state instead of the error vector of the full seven-component state, in parallel with the procedure commonly used to estimate the constrained four-component quaternion representation of attitude. Different filters in this class all use the same seven-component state but differ in their specification of the six-component error state. Two of these filters have been presented previously, and two new formulations are introduced in this paper. The sixcomponent error states of the two filters introduced here comprise the vector of infinitesimal attitude error angles and the angular momentum vector in either the spacecraft body frame or in an inertial frame.

These four filters, along with a conventional quaternion-based filter, were tested using data simulated to represent a spacecraft of the THEMIS mission. A nominal test was performed for torque-free motion, with a two degree nutation angle, no sensor misalignments, an accurately modeled inertia tensor, three-axis magnetometer data, and Sun sensor data. Three variations on this nominal test were run by adding gyro data, misaligning the magnetometer and Sun sensor by $0.1^{\circ}$, and applying a torque to precess the spin axis by $30^{\circ}$. The filter was not given the torque values for any of the tests, because that would have resulted in estimation errors indistinguishable from the nominal case. The performance of the filters was virtually identical in this series of tests, with the exception that the oldest version of the filter, SpinKF1, performed significantly better in the $30^{\circ}$ slew case. The reason for this is unknown, and the execution time of this filter was almost twice as great of that of the two filters introduced in this paper, in part because its partial derivative expressions are much more complicated, but also because its code has not been as thoroughly optimized. The five filters would have all performed equally well in the $30^{\circ}$ slew case if they had been provided with perfect torque information.

This baseline set of cases was followed by a series of tests designed to stress the filters in four different ways: reducing the number of observations per spin period, increasing the propagation step size, increasing the initial attitude error, and increasing the error in the modeled moment-of-inertia tensor. The oldest version of the filter, SpinKF1, and the new variant, SpinKF4, employing the infinitesimal attitude error angles and the angular momentum components in an inertial reference frame as the error state showed the most robust performance in these tests. On balance, the SpinKF4 version of the filter showed the best combination of robustness and efficiency in the simulations.

\section{References}

${ }^{1}$ Markley, F. L., "New Dynamic Variables for Momentum-Bias Spacecraft," The Journal of the Astronautical Sciences, Vol. 41, No. 4, pp. 557-567, 1993

${ }^{2}$ Lefferts, E. J., Markley, F. L., and Shuster, M. D., "Kalman Filtering for Spacecraft Attitude Estimation," Journal of Guidance, Control, and Dynamics, Vol. 5, No. 5, pp. 417-429, 1982

${ }^{3}$ Markley, F. L., “Attitude Estimation or Quaternion Estimation?" The Journal of the Astronautical Sciences Vol. 52, No. 1/2, pp. 221-238, 2004

${ }^{4}$ Shuster, Malcolm D., "Constraint in Attitude Estimation Part I: Constrained Estimation," The Journal of the Astronautical Sciences Vol. 51, No. 1, pp. 51-74, 2003

${ }^{5}$ Shuster, Malcolm D., "Constraint in Attitude Estimation Part II: Unconstrained Estimation," The Journal of the Astronautical Sciences Vol. 51, No. 1, pp. 75-101, 2003

${ }^{6}$ Pittelkau, M. E., "An Analysis of the Quaternion Attitude Determination Filter," The Journal of the Astronautical Sciences, Vol. 51, No. 1, pp. 103-120, 2003

${ }^{7}$ Sedlak, J. E., "Spinning Spacecraft Attitude Estimation Using Markley Variables: Filter Implementation and Results," 2005 Flight Mechanics Symposium, Goddard Space Flight Center, Greenbelt, MD, NASA Conference Publication NASA/CP-2005-212789, October 2005 
${ }^{8}$ D. Speer, G. Jackson, K. Stewart, and A. Hernandez-Pellerano, "The Space Technology 5 Avionics System," 2005 IEEE Aerospace Conference, Big Sky, MT, 05-12 March 2005

${ }^{9}$ Sedlak, Joseph, "Flight Dynamics (FD) Task Order 88, Spacecraft Attitude Determination Accuracy From Mission Experience, Revision 1, Update 6," Lanham, Maryland, December 14, 2006, Honeywell Technology Solutions Inc. Document FDF-88-021

${ }^{10}$ Markley, F. L., and Sedlak, J. E, "Kalman Filtering of Angular-Momentum-Based Attitude Parameters," in 7th Cranfield Conference on Dynamics and Control of Systems and Structures in Space, ed. by Stephen Hobbs, Cranfield University Press, Cranfield, Bedford, UK, 2006

${ }^{11}$ Frey, S., et al. "The THEMIS Mission Design," Space Science Reviews (in press)

${ }^{12}$ Wertz, J. R., ed., Spacecraft Attitude Determination and Control, Chapter 16, D. Reidel, Dordrecht, Holland, 1978

${ }^{13}$ Golub, Gene H. and Charles F. Van Loan, Matrix Computations, p. 139, The Johns Hopkins University Press, Baltimore, MD, 1983

${ }^{14} \mathrm{Gelb}$, Arthur, ed., Applied Optimal Estimation, the MIT Press, Cambridge, MA, 1974.

${ }^{15}$ Shuster, Malcolm D., "A Survey of Attitude Representations," The Journal of the Astronautical Sciences, Vol. 41, No. 4, pp. 439-517, 1993

${ }^{16}$ J. Sedlak and D. Chu, "Kalman Filter Estimation of Attitude and Gyro Bias with the QUEST Observation Model," AAS 93-297, Proceedings of the AAS/GSFC International Symposium on Space Flight Dynamics, Greenbelt, MD, 1993

${ }^{17}$ R. Alonso and M.D. Shuster, "Complete Linear Attitude-Independent Magnetometer Calibration," The Journal of the Astronautical Sciences, Vol. 50, No. 4, pp. 477-490, 2002

${ }^{18}$ Sedlak, J. E., "Iterative Magnetometer Calibration," Paper No. ALAA-2006-6386, AIAA/AAS Astrodynamics Specialist Conference, Keystone, CO, August 2006 